\title{
On application of multi-colour photometry of $\delta$ Scuti stars
}

\author{
J. Daszyńska-Daszkiewicz ${ }^{1,2}$, W.A. Dziembowski ${ }^{3,4}$, \\ A.A. Pamyatnykh ${ }^{3,5,6}$ \\ ${ }^{1}$ Astronomical Institute of the Wroctaw University, ul. Kopernika 11, \\ 51-622 Wroctaw, Poland \\ ${ }^{2}$ Instituut voor Sterrenkunde, Katholieke Universiteit Leuven, \\ Celestijnenlaan 200 B, B-3001 Leuven, Belgium \\ ${ }^{3}$ Copernicus Astronomical Center, Bartycka 18, 00-716 Warsaw, Poland \\ ${ }^{4}$ Warsaw University Observatory, Al. Ujazdowskie 4, Warsaw, Poland \\ ${ }^{5}$ Institute of Astronomy, Russian Academy of Sciences, Pyatnitskaya \\ Str. 48, 109017 Moscow, Russia \\ ${ }^{6}$ Institute of Astronomy, University of Vienna, Türkenschanzstr.17, \\ A-1180 Vienna, Austria
}

\begin{abstract}
In $\delta$ Scuti star models the photometric amplitudes and phases exhibit a strong dependence on convection, which enters through the complex parameter, $f$, that describes the bolometric flux variation. We present a new method of extracting $\ell$ and $f$ from multi-colour data and apply it to several $\delta$ Scuti stars. The inferred values of $f$ are sufficiently accurate to yield a useful constraint on models of stellar convection.
\end{abstract}

\section{Introduction}

Until now the main application of multi-colour photometry of $\delta$ Scuti stars has been the determination of the spherical harmonic degree, $\ell$, of the observed modes (e.g. Balona \& Evers 1999; Garrido 2000). The amplitudes and phases from measurements in various passbands do contain a signature of the $\ell$ value, but there is more information to be extracted from these quantities. In principle we may calculate their theoretical counterparts. For this we need appropriate stellar models and a linear nonadiabatic code for calculating model oscillations. From such calculations we derive the complex ratio of the local flux variation to the radial displacement at the photosphere, $f$. In the case of $\delta$ Scuti stars, however, there is a large uncertainty arising from lack of adequate theory of stellar convection. The uncertainty is reflected in a strong sensitivity of $f$ to the mixing-length parameter, $\alpha$, which translates to calculated mode positions in the amplitude ratio vs. phase difference diagrams. This strong sensitivity to the treatment of convection is not necessarily bad news, if we can determine simultaneously $\ell$ and $f$. For this aim we need data from at least three passbands. If we succeed, then the $f$-value yields a useful constraint on convection. In addition, if the identified mode is radial, the multi-passband data may be used to refine stellar parameters. Finally, the use of radial velocity measurements with our method significantly improves the determination of $\ell$ and $f$. 

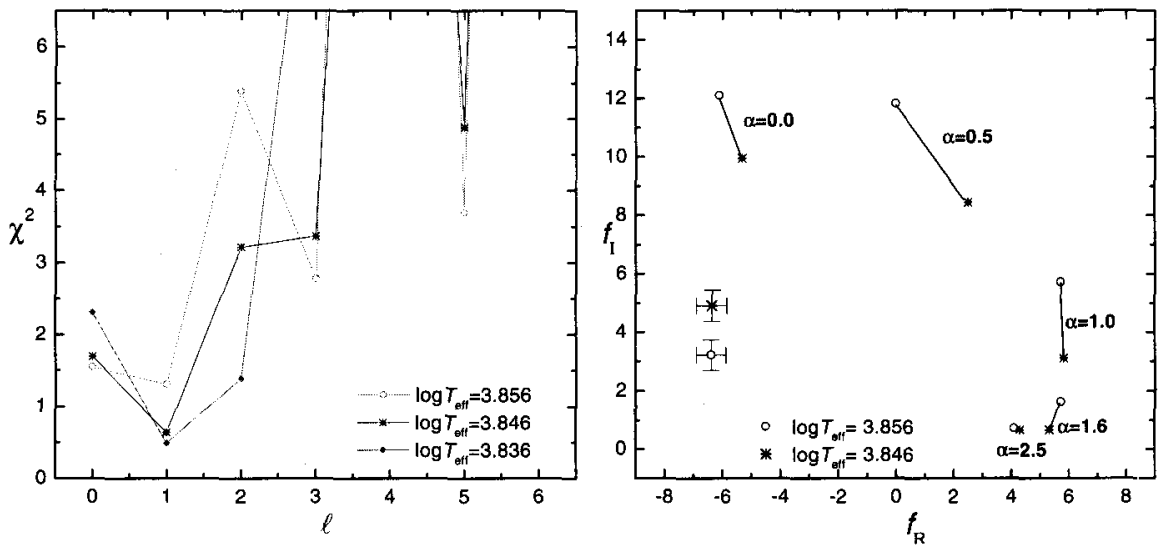

Figure 1. Left panel: $\chi^{2}$ as a function of $\ell$ for three models of $\beta$ Cas. Right panel: comparison of empirical and theoretical values of $f$ calculated for various values of $\alpha$ and two indicated effective temperatures.

\section{Method for inferring $f$ parameter from observations}

The presented method is based on $\chi^{2}$ minimization assuming trial values of $\ell$. We regard the $\ell$ degree and the associated complex $f$ value as the solution if it corresponds to a $\chi^{2}$ minimum which is much deeper than at other values of $\ell$. We write the photometric complex amplitude in the form of the linear observational equation for a number of passbands, $\lambda$,

$$
\mathcal{D}_{\ell}^{\lambda}(\tilde{\varepsilon} f)+\mathcal{E}_{\ell}^{\lambda} \tilde{\varepsilon}=A^{\lambda},
$$

where

$$
\tilde{\varepsilon} \equiv \varepsilon Y_{\ell}^{m}(i, 0), \quad \mathcal{D}_{\ell}^{\lambda} f \equiv b_{\ell}^{\lambda} D_{1, \ell}^{\lambda}, \quad \mathcal{E}_{\ell}^{\lambda} \equiv b_{\ell}^{\lambda}\left(D_{2, \ell}+D_{3, \ell}^{\lambda}\right) .
$$

On the right-hand side we have measured amplitudes, $A^{\lambda}$, expressed in the complex form. The quantities to be determined are $(\tilde{\varepsilon} f)$ and $\tilde{\varepsilon}$.

If we have data on spectral line variations, the set of equations (1) may be supplemented with an expression for the first moment, $\mathcal{M}_{1}^{\lambda}$,

$$
\mathrm{i} \omega R\left(u_{\ell}^{\lambda}+\frac{G M v_{\ell}^{\lambda}}{R^{3} \omega^{2}}\right) \tilde{\varepsilon}=\mathcal{M}_{1}^{\lambda}
$$

For more details and generalization of the method to the case of modes coupled by rotation see Daszyńska-Daszkiewicz et al. (2003).

\section{Applications}

Here we rely on Kurucz (1998) models of stellar atmospheres and Claret (2000) computations of limb-darkening coefficients. For application of the method we used uvby Strömgren photometry in all cases. 

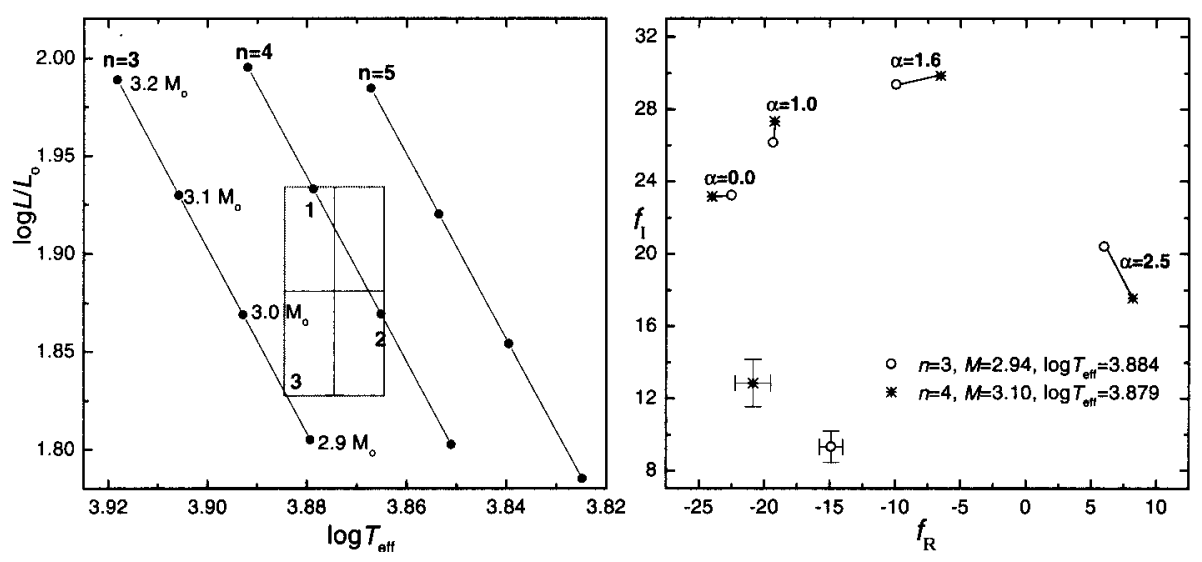

Figure 2. Left panel: an HR diagram with the observational error box for $20 \mathrm{CVn}$. Right panel: comparison of empirical values of the $f$ parameter with theoretical ones calculated for four values of $\alpha$.

$\beta$ Cas: $\beta$ Cas is a near and bright $\delta$ Scuti star, therefore we have rather precise parameters for it. Its estimated value of mass is $1.95 \mathrm{M}_{\odot}$. In the left panel of Fig. 1 we show $\chi^{2}$ as a function of $\ell$ obtained for three models. The minimum at $\ell=1$ is the deepest one, particularly at lower $T_{\text {eff }}$. Because the star is a relatively rapid rotator $\left(v_{\text {rot }}>70 \mathrm{~km} \mathrm{~s}^{-1}\right)$, we have considered the possibility that the mode is an $\ell=0$ and 2 combination resulting from the rotational coupling. We found, however, that at no inclination is the $\chi^{2}$ value as low as at a single $\ell=1$. Thus, the latter identification is most likely.

In the right panel we show a comparison of the $f$ values inferred from observations for $\beta$ Cas with the theoretical values calculated with the five indicated values of the MLT parameter, $\alpha$. The observed values of $f_{R}$ are closer to those calculated with $\alpha=0$, but values of $f_{I}$ require rather higher values of $\alpha$.

$20 \mathrm{CVn}: 20 \mathrm{CVn}$ is a $\delta$ Scuti variable regarded to be monoperiodic with a metal abundance of $[\mathrm{m} / \mathrm{H}]=0.5(Z \approx 0.06)$. For models on the edges of the error box at $Z=0.06$, the minimum of the $\chi^{2}$ is clearly at $\ell=0$. Having such a mode identification we can refine stellar parameters by fitting them to reproduce the observed period.

In the HR diagram shown in the left panel of Fig. 2, we plot the error box and the lines of the constant period for radial orders $n=3,4,5$ at $Z=0.06$. Only models along these lines are allowed. We can see that we have two possibilities: $n=3$ or $n=4$. The models yielding the lowest $\chi^{2}$ are those marked with numbers 1 and 3 , both equally probable. In the case of $Z=0.04$ only $n=4$ is allowed, but the $\chi^{2}$ is much higher. Thus from our method we also have some constraints on metallicity. In the right panel we compare the empirical and theoretical values of the $f$ parameter for models corresponding to the deepest $\chi^{2}$ minima. The positions are qualitatively similar to those in $\beta$ Cas, but are considerably higher as a consequence of higher radial order. 

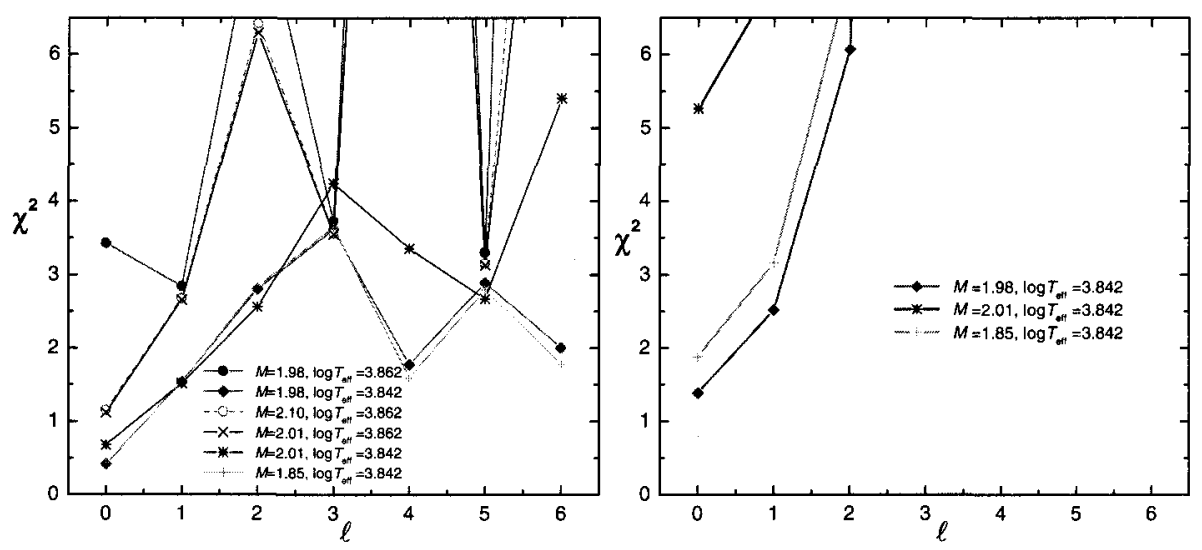

Figure 3. Left panel: $\chi^{2}$ as a function of $\ell$ from photometry of 1 Mon. Right panel: the same after including the radial velocity.

1 Mon: 1 Mon has three almost equally spaced frequencies. Here we present results for the dominant mode. In the left panel of Fig. 5 we show $\chi^{2}$ as a function of $\ell$ obtained from $u v b y$ photometry for the models on the edges of error box. In the right panel we have the same, but after including data on radial velocity changes. Discrimination is now much better. Especially the higher values of $\ell$ and some sets of stellar parameters are clearly excluded. The empirical values of the $f$ parameter are now very close to those calculated with $\alpha=0.0$. We obtained $f_{\text {obs }}=(-4.9 \pm 0.7,11.3 \pm 0.8)$ and $f_{\text {calc }}=(-2.4,11.2)$.

\section{Conclusions}

We have argued that multi-colour photometry of $\delta$ Scuti stars may give us useful information not only about the excited modes, but also about the stars themselves. We believe that the most interesting result of this is the prospect of probing the efficiency of convective transport in the outer layers. We also showed that inference on mode degree and stellar properties is greatly improved by supplementing photometric data with radial velocity measurements.

Acknowledgments. The KBN grant No. 5 P03D 01220 is acknowledged.

\section{References}

Balona, L.A., Evers, E.A. 1999, MNRAS, 302, 349

Claret, A. 2000, A\&A, 363, 1081

Daszyńska-Daszkiewicz, J., Dziembowski, W.A., Pamyatnykh, A.A. 2003, A\&A, 407,999

Garrido, R. 2000, ASP Conf. Ser., Vol. 210, Delta Scuti and Related Stars, eds. M. Breger \& M.H. Montgomery, (San Francisco ASP), 67

Kurucz, R.L. 1998, http://cfaku5.harvard.edu 